\title{
Numerical analysis of spatial evolution of the small signal gain in a chemical oxygen-iodine laser operating without primary buffer gas
}

\author{
Zongmin $\mathrm{Hu}^{\mathrm{a}, \mathrm{b}}$, Zonglin Jiang ${ }^{\mathrm{a}}$, Rhoshin Myong ${ }^{\mathrm{b}, *}$, Taehwan Cho \\ ${ }^{\mathrm{a}}$ LHD Laboratory, Institute of Mechanics, CAS, Beijing 100080, China \\ ${ }^{\mathrm{b}}$ Research Center for Aircraft Parts Technology and School of Mechanical and Aerospace Engineering, \\ Gyeongsang National University, Jinju 660-701, South Korea
}

Received 14 December 2006; received in revised form 1 April 2007; accepted 2 April 2007

Available online 22 May 2007

\begin{abstract}
A chemical oxygen-iodine laser (COIL) that operates without primary buffer gas has become a new way of facilitating the compact integration of laser systems. To clarify the properties of spatial gain distribution, three-dimensional (3-D) computational fluid dynamics (CFD) technology was used to study the mixing and reactive flow in a COIL nozzle with an interleaving jet configuration in the supersonic section. The results show that the molecular iodine fraction in the secondary flow has a notable effect on the spatial distribution of the small signal gain. The rich iodine condition produces some negative gain regions along the jet trajectory, while the lean iodine condition slows down the development of the gain in the streamwise direction. It is also found that the new configuration of an interleaving jet helps form a reasonable gain field under appropriate operation conditions.
\end{abstract}

(C) 2007 Elsevier Ltd. All rights reserved.

Keywords: Chemical oxygen-iodine laser (COIL); Without buffer gas; Interleaved jet

\section{Introduction}

A chemical oxygen-iodine laser (COIL) is a shortwavelength, high-power chemical laser that operates on an atomic iodine laser transition. The ground-state iodine atom, I, is pumped into the excited state, I*, through a process of near-resonant energy transfer between metastable singlet oxygen, $\mathrm{O}_{2}\left({ }^{1} \Delta\right)$, and $\mathrm{I}$. The laser medium, $\mathrm{I}^{*}$, then radiates a photon with a wavelength of $\lambda=1.315 \mu \mathrm{m}$, which can amplify the seed beam into a high-power infrared laser beam through the resonator. Research on COILs has recently accelerated due to the potential military and industrial applications that are dedicated to systematic integration of COILs into compact mobile platforms. To meet the requirements of such integration, we must reduce the enormous volumes and weights of COIL devices while maintain the original performance. In

\footnotetext{
*Corresponding author. Research Center for Aircraft Parts Technology, Gyeongsang National University, Jinju 660-701, South Korea. Tel.: + 82557516107 ; fax: +82557575622 .

E-mail address: myong@gnu.ac.kr (R. Myong).
}

spite of the apparent difficulty of the task, it is an interesting research topic.

Recent research indicates that a COIL operating without primary buffer gas achieves higher efficiency and stability and more applicability for mobile integration [1-4]. In such a COIL system, the traditional pressure recovery device, which is driven by combustion gas injectors, is replaced with quiet cryosorption pumping equipment. However, one of the problems with this kind of COIL is that the injection orifices must be arranged downstream the nozzle throat due to the low character velocity, because this COIL device uses nitrogen rather than helium as the buffer gas [1-4]. Recently, at Ben-Gurion University [3-6], a series of experimental studies were completed with a special slit-nozzle supersonic COIL operating without primary buffer gas. Those studies concluded that the gain distribution is often inhomogeneous because of inefficient mixing [6]. Transverse jets in the transonic or supersonic section chock the nozzle flow or generically induce complicated flow discontinuities, which may in turn seriously affect the development of the mixing and chemical reaction flow. 
Enlightened by research from above literature [3-6], further study was conducted to illuminate the performance of the COIL nozzle. We introduced a new jet scheme called the interleaved jet and applied it to the COIL mixing nozzle. Using the three-dimensional (3-D) computational fluid dynamics (CFD) technology, we analyzed the nozzle flows to obtain a greater understanding of the physical processes that underlie the operation of a COIL without primary buffer gas. We focused in particular on the reactive flowfield coupling between the gas dynamics and chemical reaction kinetics, as well as the spatial evolution of the small signal gain.

\section{Coupling between the chemical reaction kinetics and gas dynamics}

In general, the gas-phase chemistry in the mixing nozzle can be described as one of three processes: the dissociation of $I_{2}$, the pumping reaction that produces an excited iodine atom, and various competing or de-active reactions. Although some uncertainties remain in the applicable kinetics rate equations and corresponding rates, predicting the dissociation of molecular iodine, $\mathrm{I}_{2}$, in an excited oxygen, $\mathrm{O}_{2}\left({ }^{1} \Delta\right)$, stream is the largest source of error in modeling the performance of COILs [7,8]. The main kinetics processes are illustrated in Fig. 1. The reactions (1)-(4) model the self-catalyzed chain reaction associated with the dissociation of $I_{2}$. Of these equations, Eq. (1) is the primary onset reaction and Eq. (3) represents the resonant energy transfer or pumping reaction through which the laser media are generated. The reactions (5), (6) and (7) are different quench processes by third bodies. Eq. (8) represents the stimulated laser radiation. During the starting stage of the dissociation process, there is insufficient I* in the flowfield to trigger and maintain reaction (4); thus, the initial rate of dissociation is controlled by Eqs. (1) and (2). As the iodine atoms are formed and pumped rapidly to the excited state via Eq. (3), the dissociation rate is consequently accelerated by the reaction (4). Once

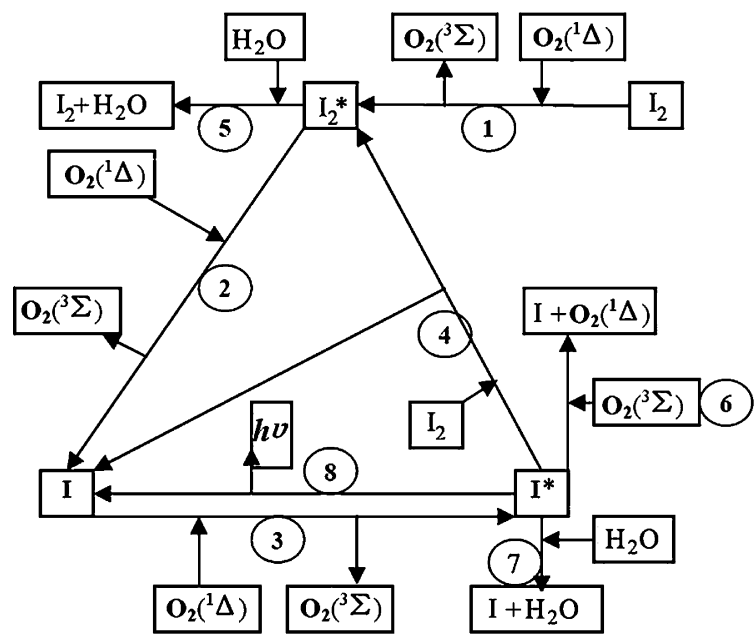

Fig. 1. Sketch of the kinetic processes in the COIL nozzle. sufficient I atoms are available to be pumped via Eq. (3), the dominating steps are handed off from reactions (1) and (2) to reactions (4) and (2). For simplicity, other reactions such as the minor onset reactions of the dissociation are not included in Fig. 1 but can be found in [9-11].

If reactions (1), (2), and (3) were the only equations working for the $\mathrm{I}_{2}$ dissociation process, the most efficient mixing scheme would rapidly mix the singlet oxygen and iodine streams to achieve a uniform mixture, thereby producing a uniform gain field. However, due to the acceleration of the dissociation process in Eq. (4), the slower mixing schemes, which can generate regions of high-molecular iodine concentration, may facilitate faster and more complete dissociation locally. At the same time, the flows should be mixed completely once the molecular iodine is fully dissociated; otherwise the overfeeding of $\mathrm{I}_{2}$ can quench $\mathrm{I}^{*}$ when $\mathrm{O}_{2}\left({ }^{1} \Delta\right)$ is consumed in the mixing flow. This process, which is the coupling process between the chemical reaction and gas dynamics, has a substantial effect on the evolution of the small signal gain in the mixing nozzle of the COIL.

\section{Numerical analysis of the flow field in a COIL that operates without primary buffer gas}

\subsection{Governing equations and numerical methods}

A COIL flow with a low density and Reynolds number from $10^{2}$ (based on the jet flow conditions and the orifice) to $10^{3}$ (based on the primary flow conditions and the inlet chamber size) is normally supposed to be governed by 3-D Navier-Stokes equations. In Cartesian coordinates, the conservative form of the $\mathrm{N}-\mathrm{S}$ equation is written as

$\frac{\partial U}{\partial t}+\frac{\partial F_{i}}{\partial x_{i}}=\frac{\partial D_{i}}{\partial x_{i}}+S(i=1,2,3)$.

Here, $U$ is the unknown variable vector, $F_{i}$ are the inviscid flux vectors in $x_{i}$ directions, $D_{i}$ are the diffusion flux vectors, and $S$ is the chemical reaction source term, where

$$
\begin{aligned}
& U=\left[\rho_{1} \rho_{2} \ldots \rho_{n s} \rho u_{1} \rho u_{2} \rho u_{3} \rho e\right], \\
& F_{i}=\left[\rho_{1} u_{i} \rho_{2} u_{i} \cdots \rho_{n s} u_{i} \rho u_{i} u_{2}+\delta_{i, 1} p \rho u_{i} u_{2}\right. \\
& \left.+\delta_{i, 2} p \rho u_{i} u_{3}+\delta_{i, 3} p(\rho e+p) u_{i}\right] \quad \text {, } \\
& D_{i}=\left[\rho_{1} v_{D 1, i} \rho_{2} v_{D 2, i} \ldots \rho_{n s} v_{D n s, i} \tau_{i, 1} \tau_{i, 2} \tau_{i, 3} u_{j} \tau_{i, j}+q_{i}\right] \\
& j=1,2,3
\end{aligned}
$$

$S=\left[\begin{array}{lllllll}\dot{\omega}_{1} & \dot{\omega}_{2} & \ldots & \dot{\omega}_{n s} & 0 & 0 & 0\end{array}\right]$.

In above equations, the total density of the mixture, $\rho=\sum_{1}^{n s} \rho_{s p}$, and the partial density of the species $s p$ $(s p=1,2, \ldots n s)$ is denoted by $\rho_{s p} . u_{i}(i=1,2,3)$ are velocity components in $x, y$ and $z$ directions. $e$ and $p$ denote the total energy and the pressure of the mixture. $V_{D s p, i}$ represents the diffusion velocity of the species $s p$ in $x_{i}$ 
direction. $\tau$ and $q$ define the viscous stress and heat conduction terms, and $\dot{\omega}_{s p}$ is the variation rate of the species $s p$. The transport properties of each pure component, such as viscosity and thermal conductivity, and the thermodynamic properties, such as the special heat capacity and enthalpy, were obtained by using the relations given in the power series form of the mixture temperature. The effective binary diffusion model rather than Fick's model was applied to approximate the diffusion velocities of all species. For the chemical kinetic model, 21 elementary reactions and the following 10 species: $\mathrm{I}_{2}, \mathrm{I}_{2}$ *, I, I*, $\mathrm{O}_{2}\left({ }^{1} \Delta\right), \mathrm{O}_{2}\left({ }^{1} \Sigma\right), \mathrm{O}_{2}\left({ }^{3} \Sigma\right), \mathrm{H}_{2} \mathrm{O}, \mathrm{Cl}_{2}$ and $\mathrm{N}_{2}$, were considered. The reaction set is given in Table 1 in detail, which synthesizes data from [9-11]. Note that the reaction numbers in Table 1 may not consist with those in Fig. 1. A synthetic analysis of the transport property model, thermodynamics definitions, diffusion model, and chemical reactions was presented in [9-12].

The semi-discretized difference equation for Eq. (1) is

$$
\frac{\partial U}{\partial t}=-\mathrm{CONV}_{i, j, k}^{n}+\operatorname{VISC}_{i, j, k}^{n}+S_{i, j, k}^{n} \text {. }
$$

The dissipative term, VISC, is discretized by a secondorder centered difference scheme, while the convective term, CONV, is discretized with the dispersion-controlled dissipative (DCD) scheme [13,14] proposed by Jiang et al. [13]. The DCD scheme has been shown to be simple and robust means of capturing shock waves. The computational domains used in present simulations were all discretized on body-fitted, structured grids, with local mesh refinement near the jet regions and the solid boundaries. An explicit and second-order Runge-Kutta integration method is used to advance the computational solution in time toward steady state. The convergence

Table 1

Reaction set (synthesis of data from [9-11])

\begin{tabular}{llc}
\hline Number & Reaction & $k_{\mathrm{f}}\left(\mathrm{cm}^{3}\right.$ molecule $\left.{ }^{-1} \mathrm{~s}^{-1}\right)$ \\
\hline 1 & $\mathrm{O}_{2}\left({ }^{1} \Delta\right)+\mathrm{O}_{2}\left({ }^{1} \Delta\right) \rightarrow \mathrm{O}_{2}\left({ }^{1} \Sigma\right)+\mathrm{O}_{2}\left({ }^{3} \Sigma\right)$ & $2.7 \times 10^{-17}$ \\
2 & $\mathrm{O}_{2}\left({ }^{1} \Sigma\right)+\mathrm{H}_{2} \mathrm{O} \rightarrow \mathrm{O}_{2}\left({ }^{1} \Delta\right)+\mathrm{H}_{2} \mathrm{O}$ & $6.7 \times 10^{-12}$ \\
3 & $\mathrm{O}_{2}\left({ }^{1} \Delta\right)+\mathrm{O}_{2}\left({ }^{3} \Sigma\right) \rightarrow \mathrm{O}_{2}\left({ }^{3} \Sigma\right)+\mathrm{O}_{2}\left({ }^{3} \Sigma\right)$ & $1.6 \times 10^{-18}$ \\
4 & $\mathrm{O}_{2}\left({ }^{1} \Delta\right)+\mathrm{H}_{2} \mathrm{O} \rightarrow \mathrm{O}_{2}\left({ }^{3} \Sigma\right)+\mathrm{H}_{2} \mathrm{O}$ & $4.0 \times 10^{-18}$ \\
5 & $\mathrm{O}_{2}\left({ }^{1} \Delta\right)+\mathrm{Cl}_{2} \rightarrow \mathrm{O}_{2}\left({ }^{3} \Sigma\right)+\mathrm{Cl}_{2}$ & $6.0 \times 10^{-18}$ \\
6 & $\mathrm{I}_{2}+\mathrm{O}_{2}\left({ }^{1} \Sigma\right) \rightarrow 2 \mathrm{I}+\mathrm{O}_{2}\left({ }^{3} \Sigma\right)$ & $4.0 \times 10^{-12}$ \\
7 & $\mathrm{I}_{2}+\mathrm{O}_{2}\left({ }^{1} \Sigma\right) \rightarrow \mathrm{I}_{2}+\mathrm{O}_{2}\left({ }^{3} \Sigma\right)$ & $1.6 \times 10^{-11}$ \\
8 & $\mathrm{I}_{2}+\mathrm{O}_{2}\left({ }^{1} \Delta\right) \rightarrow \mathrm{I}_{2}{ }^{*}+\mathrm{O}_{2}\left({ }^{3} \Sigma\right)$ & $7.0 \times 10^{-15}$ \\
9 & $\mathrm{I}_{2}+\mathrm{I}^{*} \rightarrow \mathrm{I}+\mathrm{I}_{2}{ }^{*}$ & $3.5 \times 10^{-11}$ \\
10 & $\mathrm{I}_{2}{ }^{*}+\mathrm{O}_{2}\left({ }^{1} \Delta\right) \rightarrow 2 \mathrm{I}+\mathrm{O}_{2}\left({ }^{3} \Sigma\right)$ & $3.0 \times 10^{-10}$ \\
11 & $\mathrm{I}_{2}{ }^{*}+\mathrm{O}_{2}\left({ }^{3} \Sigma\right) \rightarrow \mathrm{I}_{2}+\mathrm{O}_{2}\left({ }^{3} \Sigma\right)$ & $1.7 \times 10^{-12}$ \\
12 & $\mathrm{I}_{2}{ }^{*}+\mathrm{H}_{2} \mathrm{O} \rightarrow \mathrm{I}_{2}+\mathrm{H}_{2} \mathrm{O}$ & $8.2 \times 10^{-12}$ \\
13 & $\mathrm{I}_{2}{ }^{*}+\mathrm{N}_{2} \rightarrow \mathrm{I}_{2}+\mathrm{N}_{2}$ & $5.0 \times 10^{-18}$ \\
14 & $\mathrm{I}^{*}+\mathrm{N}_{2} \rightarrow \mathrm{I}+\mathrm{N}_{2}$ & $7.8 \times 10^{-11}$ \\
15 & $\mathrm{I}+\mathrm{O}_{2}\left({ }^{1} \Delta\right) \rightarrow \mathrm{I}^{*}+\mathrm{O}_{2}\left({ }^{3} \Sigma\right)$ & $1.0 \times 10^{-15}$ \\
16 & $\mathrm{I}^{*}+\mathrm{O}_{2}\left({ }^{3} \Sigma\right) \rightarrow \mathrm{I}+\mathrm{O}_{2}\left({ }^{1} \Delta\right)$ & $1.0 \times 10^{-13}$ \\
17 & $\mathrm{I}+\mathrm{O}_{2}\left({ }^{1} \Delta\right) \rightarrow \mathrm{I}+\mathrm{O}_{2}\left({ }^{3} \Sigma\right)$ & $1.1 \times 10^{-13}$ \\
18 & $\mathrm{I}^{*}+\mathrm{O}_{2}\left({ }^{1} \Delta\right) \rightarrow \mathrm{I}+\mathrm{O}_{2}\left({ }^{1} \Sigma\right)$ & $1.7 \times 10^{-13}$ \\
19 & $\mathrm{I}^{*}+\mathrm{O}_{2}\left({ }^{1} \Delta\right) \rightarrow \mathrm{I}+\mathrm{O}_{2}\left({ }^{1} \Delta\right)$ & $2.1 \times 10^{-12}$ \\
20 & $\mathrm{I}^{*} \mathrm{I}^{*} \rightarrow \mathrm{I}+\mathrm{I}$ & \\
21 & $\mathrm{I}^{*}+\mathrm{H}_{2} \mathrm{O} \rightarrow \mathrm{I}+\mathrm{H}_{2} \mathrm{O}$ & \\
\hline
\end{tabular}

criterion is defined as Res $=\left(\dot{m}_{\text {in }}-\dot{m}_{\text {out }}\right) / \dot{m}_{\text {in }} \leqslant 10^{-4}$, where $\dot{m}_{\text {in }}$ and $\dot{m}_{\text {out }}$ are the mass flow rates of some important species at the inflow and outflow surface of the nozzle, respectively; for example, helium in the validation case and overall oxygen in other cases. One run took about a month on a self-parallelized 64-bit workstation with 4-processors.

\subsection{Validation of the numerical simulations}

To verify the numerical algorithms and validate the numerical solutions, a cold flow with a transonic injection in a RADICL (research assessment device improvement chemical laser) slit nozzle has been studied. The geometry used in the simulation was based on the experimental geometry used in [15]. The computational domain used half
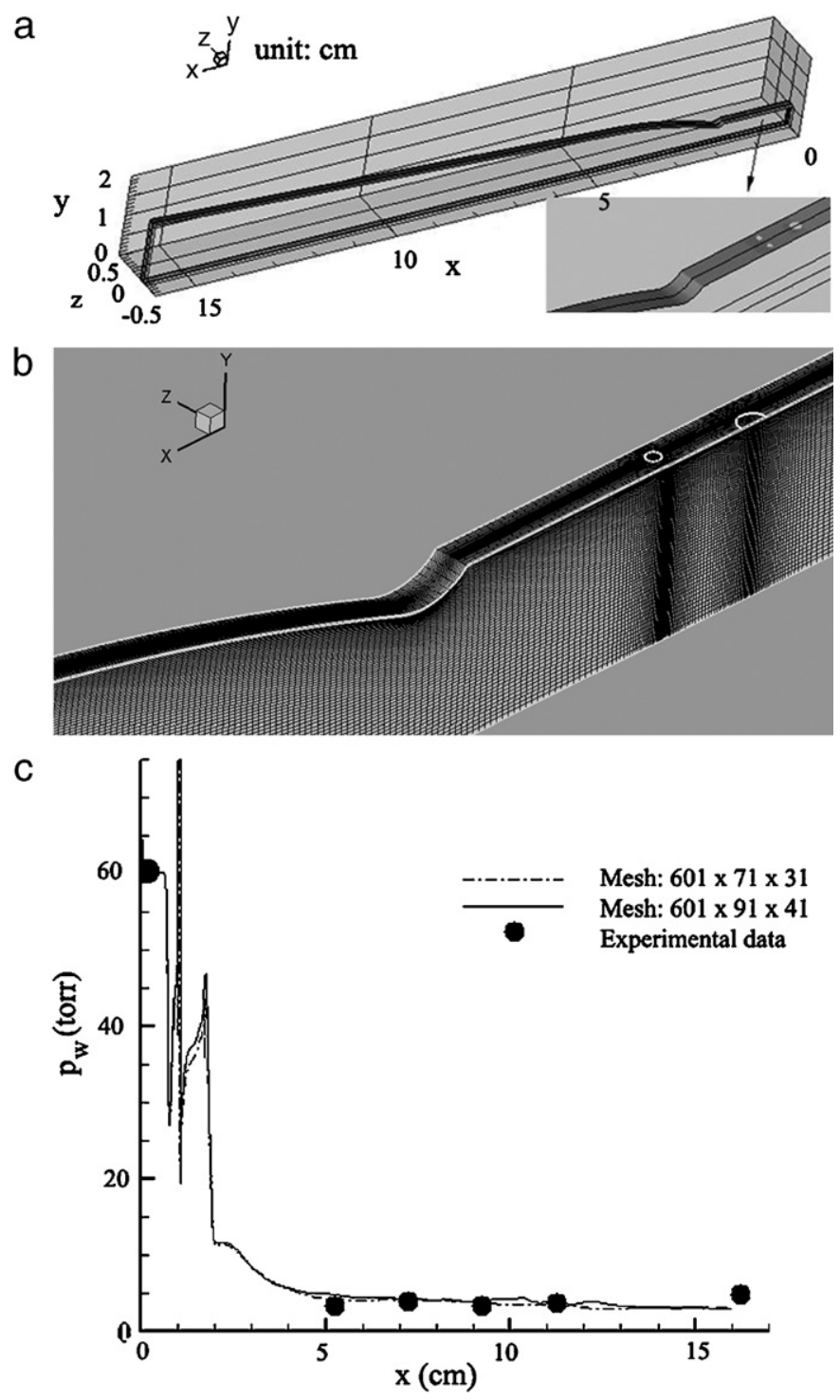

Fig. 2. RADICL slit nozzle flow simulation for validation and gridindependence analysis: (a) Computational domain, (b) Mesh close-up near the jet region, and (c) Comparison between the numerical results and experimental data in wall pressure. 
of the unit cell approximation, which is based on the symmetry of the RADICL nozzle geometry and the symmetry hypothesis of the multi-jet flow. Fig. 2a shows the unit cell. A coarse grid $(601 \times 71 \times 31)$ as shown in Fig. $2 b$ and a fine grid $(601 \times 91 \times 41)$ were used to discretize the nozzle. The flow conditions were set according to the experimental setup in [15]. First, a secondary flow was perpendicularly injected into the primary flow near the nozzle throat. Second, helium was used for both the primary and secondary flows. Last, we used a total temperature and pressure of $T_{\mathrm{p}}=293 \mathrm{~K}$ and $p_{\mathrm{p}}=7955 \mathrm{~Pa}$ for the primary flow $(\mathrm{p})$, and $T_{\mathrm{s}}=403 \mathrm{~K}$ and $p_{\mathrm{s}}=32925 \mathrm{~Pa}$ for the secondary flow $\left({ }_{\mathrm{s}}\right)$. For the boundary condition in this calculation, the sonic inflow condition was set to the secondary injection, while the constant total pressure condition was imposed on the subsonic inflow plane for the primary flow.

Fig. 2c shows computed wall pressure together with the measured experimental data. There is a good agreement between the numerical results for both the coarse and fine grids and the experiment. The discrepancy in the pressure measurements is highest near the downstream nozzle exit, which is considered to be a consequence of injecting a purge flow in the $z$ direction downstream of the nozzle throat in the experiment [15]. The application of the fine grid causes a minor change in the wall pressure. From a direct comparison of these results, it can be concluded that the numerical solutions are well validated for the jet flow in the COIL.

\subsection{Computational setup}

Downstream the throat, two rows of jet orifices are located on either side of the nozzle blade. The jet orifices, which have a diameter of $1 \mathrm{~mm}$, are interleaved and set $2 \mathrm{~mm}$ apart from each other in the $z$ direction. The height of the nozzle throat is $6 \mathrm{~mm}$. The angle between the secondary jets and the nozzle axis is $45^{\circ}$. Fig. 3 demonstrate the nozzle bank, the interleaved jet arrangement, the simplified computational domain which includes a half-jet
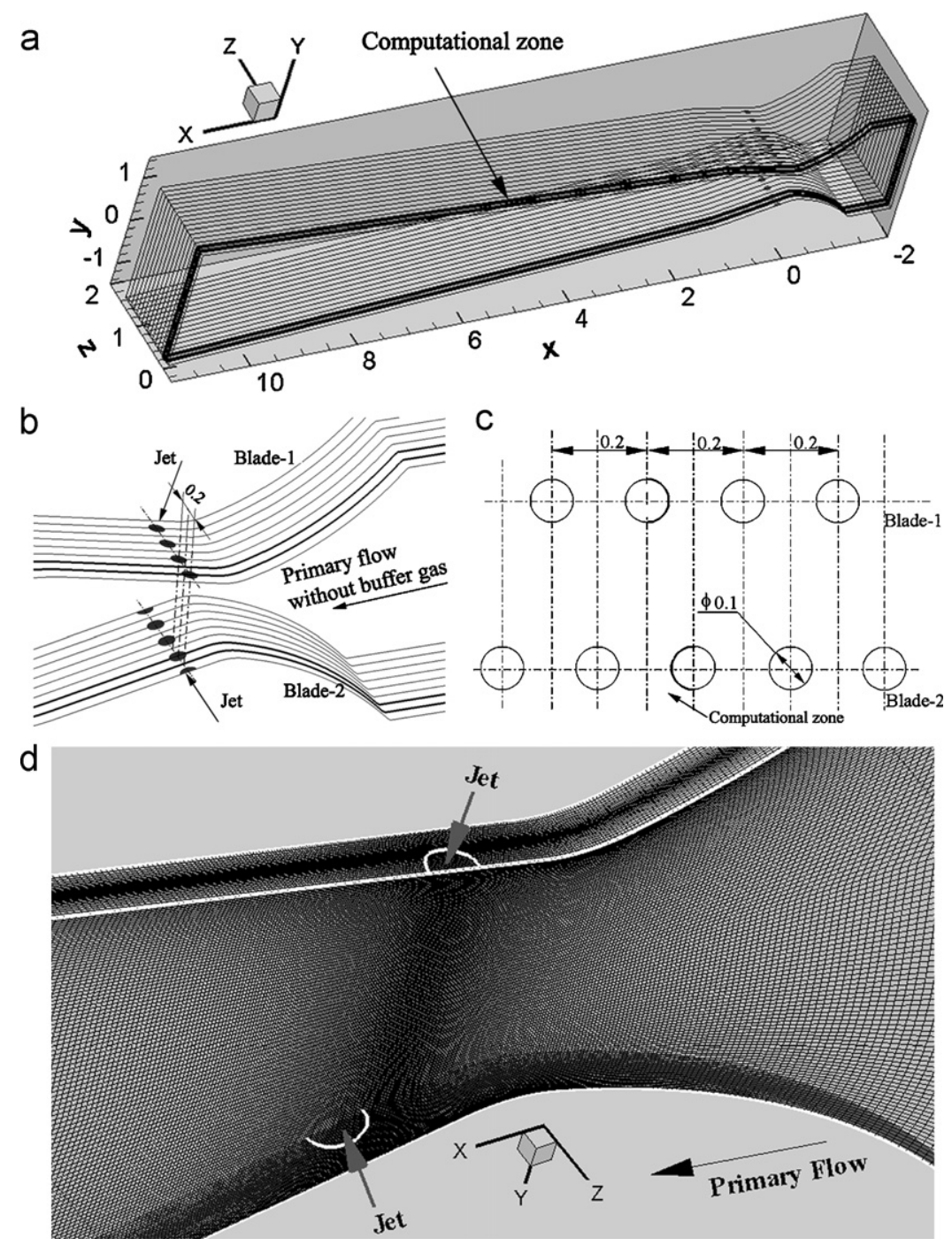

Fig. 3. (a) Computational domain for the interleaved jet mixing, (b) Close-up of the jet region, (c) The arrangement of the jet orifice, and (d) Mesh closeup near the jet region (Length unit: $\mathrm{cm}$ ). 


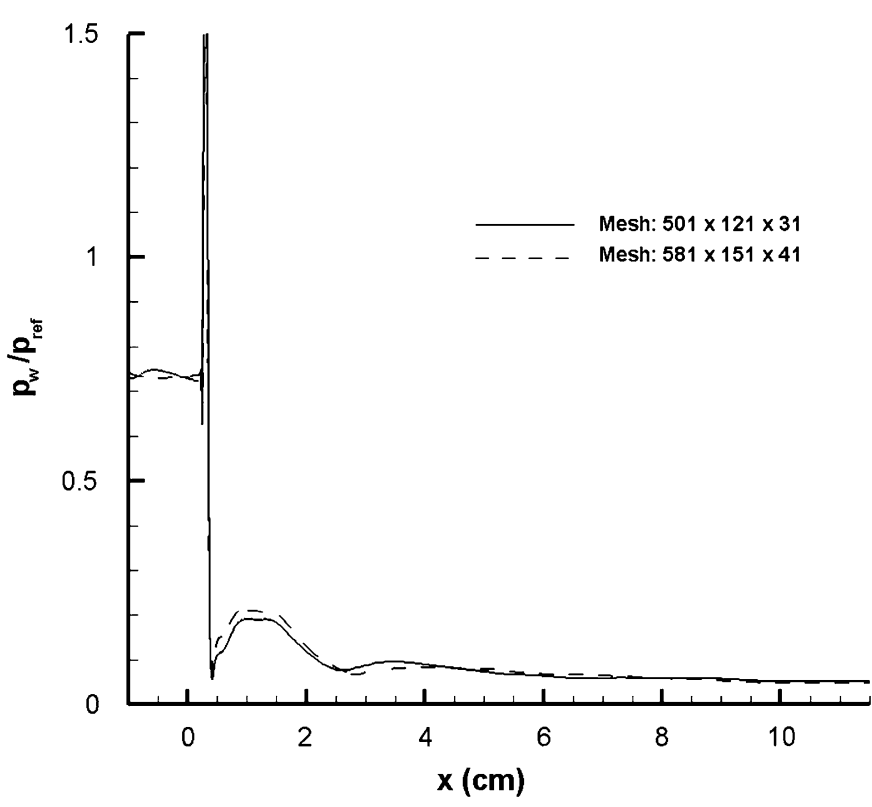

Fig. 4. Grid convergence for the interleaved jet geometry.
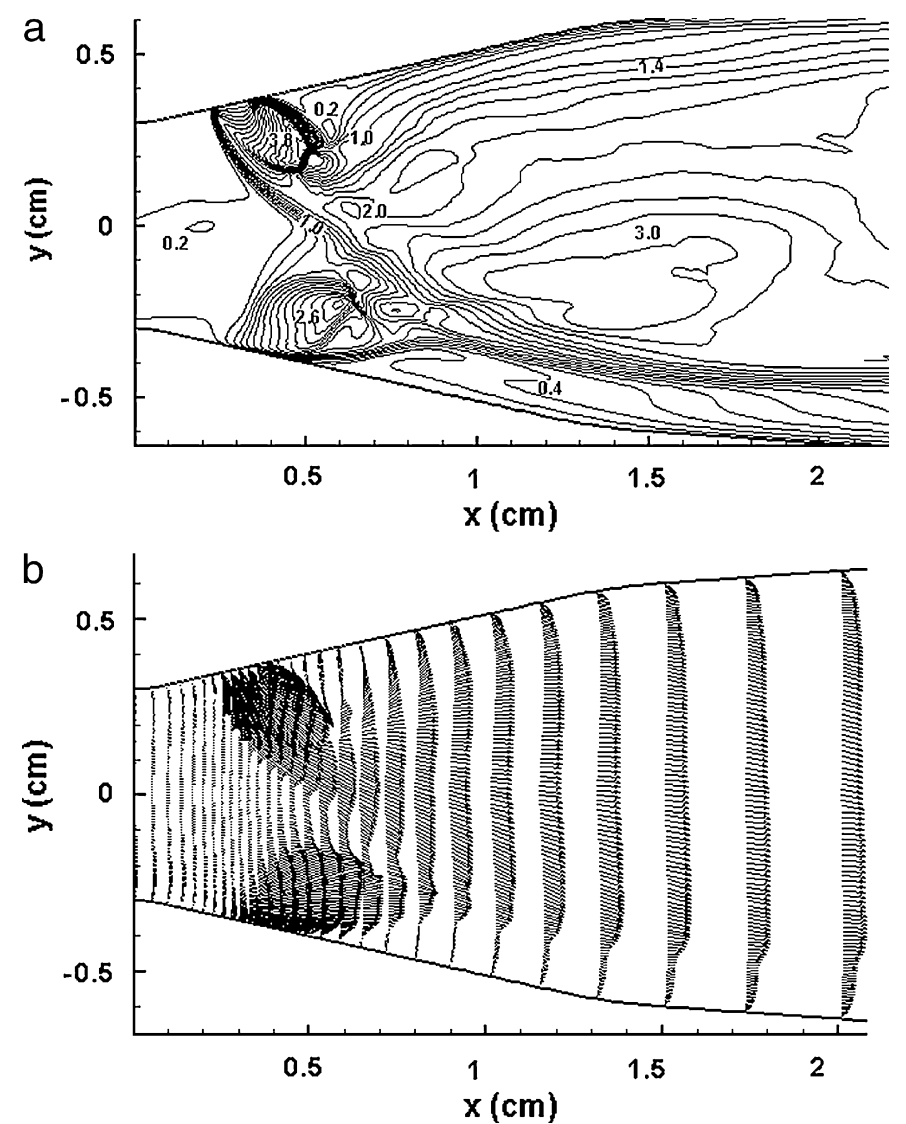

Fig. 5. Flow structures in the near field of jet: (a) Mach number and (b) Two-dimensional flow vector in the symmetric plane of the upper jet orifice (the flow direction is from left to right).

orifice on the either side of the nozzle blade, and the mesh enlargement near the jet region. The secondary flow is diluted by the buffer gas $\mathrm{N}_{2}$. Three cases were considered, each with a different molar ratio of $\mathrm{I}_{2} / \mathrm{N}_{2}$ : namely $1 / 60$,
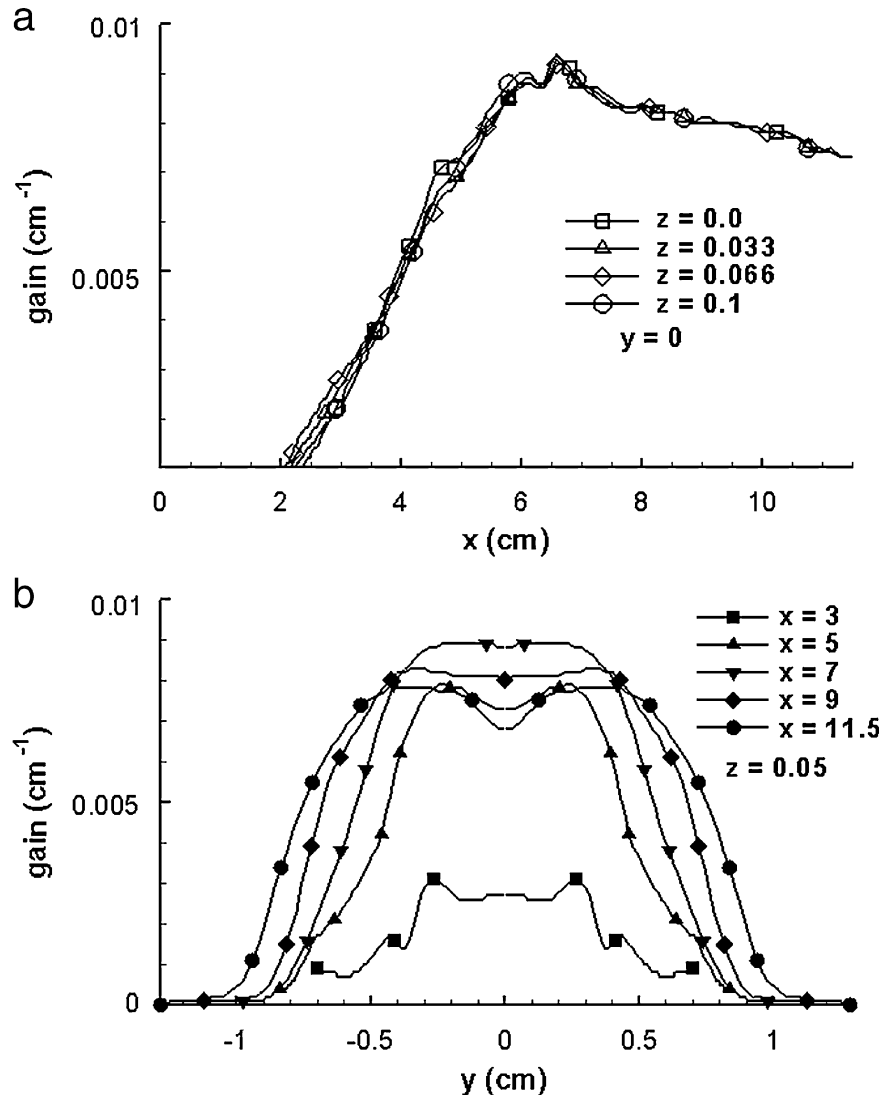

Fig. 6. Distribution of the small signal gain (for case (1) $I_{2} / N_{2}=1 / 60$ ): (a) Along the $x$ direction (in the $y=0$ plane) and (b) Along the $y$ direction (in the $z=0.05$ plane).

$2 / 59$, and $0.5 / 60.5$. The total flow rate of the secondary jets, $\dot{n}_{s}$, is $1.86 \times 10^{-5} \mathrm{~mol} \mathrm{~s}^{-1}$, and the stagnation temperature, $T_{\mathrm{s} 0}$, is $383 \mathrm{~K}$. For all cases considered, the molar ratio of the primary flow, $\mathrm{O}_{2}\left({ }^{3} \Sigma\right) / \mathrm{O}_{2}\left({ }^{1} \Delta\right) / \mathrm{H}_{2} \mathrm{O} / \mathrm{Cl}_{2}$, is given as $1 / 1.5 /$ $0.278 / 0.278$. The primary flow rate, $\dot{n}_{\mathrm{p}}$, is $0.99 \times$ $10^{-5} \mathrm{~mol} \mathrm{~s}^{-1}$, while $T_{\mathrm{p} 0}=271.3 \mathrm{~K}$.

Total grid point of $501 \times 121 \times 31$ is used to discretize the studied geometry, while mesh refinement is handled near the jets and the nozzle walls. For grid convergence, another grid with $581 \times 151 \times 41$ nodes was use to simulate the first case. The normalized wall pressure along the centerline of the jet orifice was given in Fig. 4. Two solutions are nearly consistent with each other. The coarse gird was chosen to study the interleaved jet geometry.

\subsection{Features of the flowfield}

In conditions where nitrogen buffer gas or no primary buffer gas is used, it is reasonable to move the secondary jet orifices downstream to the transonic or supersonic section of the mixing nozzle. Generally, the injection of gas into the supersonic flow induces bow shock waves in front of the jet trajectories. If the structures block the stream to a certain extent, the geometry throat does not work and a gas dynamic throat takes over the responsibility of the subsonic-supersonic transition. Fig. 5 shows the flowfield 

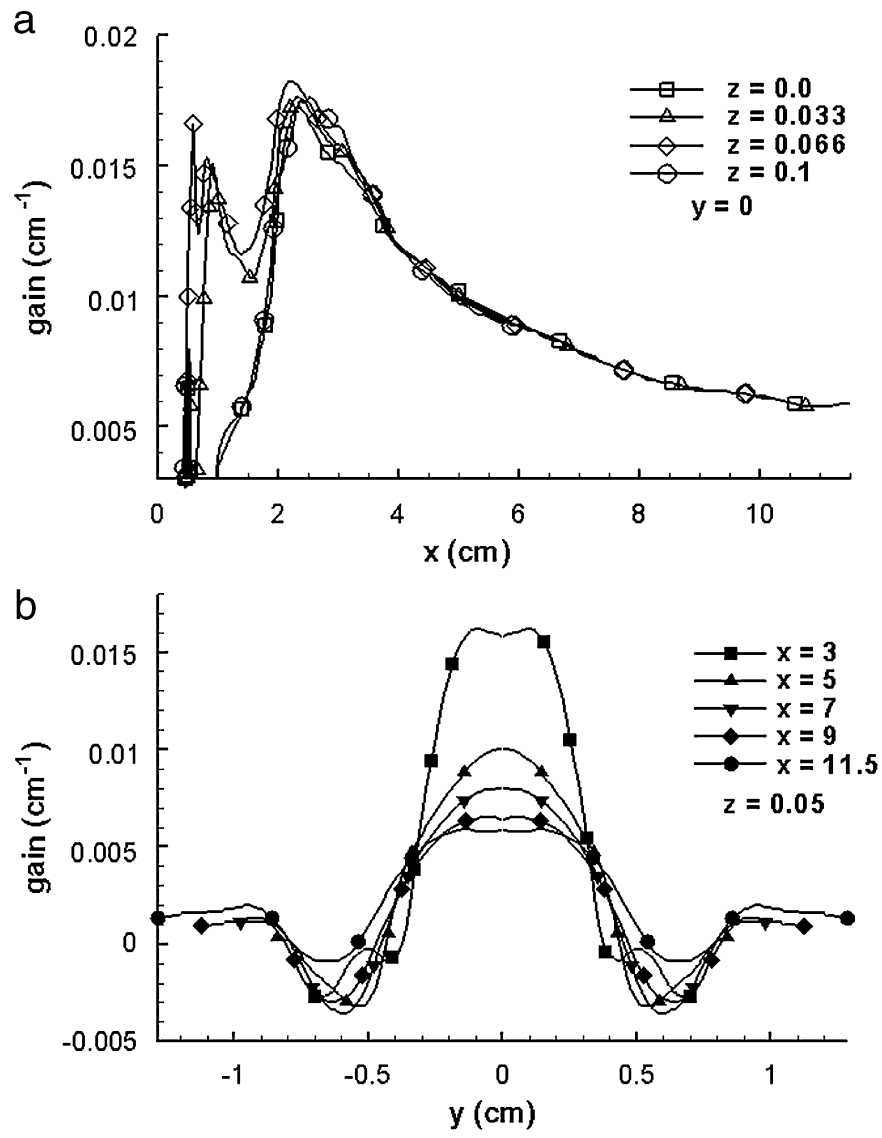

Fig. 7. Distribution of the small signal gain (for case (2) $I_{2} / N_{2}=2 / 59$ ): (a) Along the $x$ direction (in the $y=0$ plane) and (b) Along the $y$ direction (in the $z=0.05$ plane).

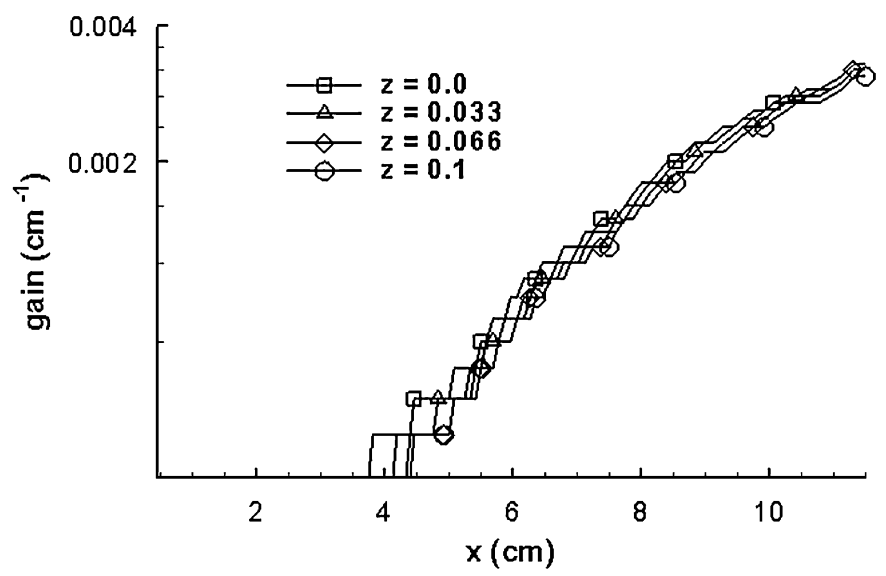

Fig. 8. Distribution of the small signal gain when iodine is not fed sufficiently (for case (3) $\mathrm{I}_{2} / \mathrm{N}_{2}=0.5 / 60.5$ ).

in the $z=0$ symmetric plane of the upper jet orifice. In Fig. 5a, an irregular gas dynamic throat can be seen, as well as a barrel shock and a Mach disk associated with the under-expanded jet condition. The interleaved jets result in an asymmetric flowfield with the sharp gradients of the velocity, temperature, and species concentration in the near field of the jets. As shown in Fig. 5b, a secondary recirculation exists in the wake of the upper jet flow.
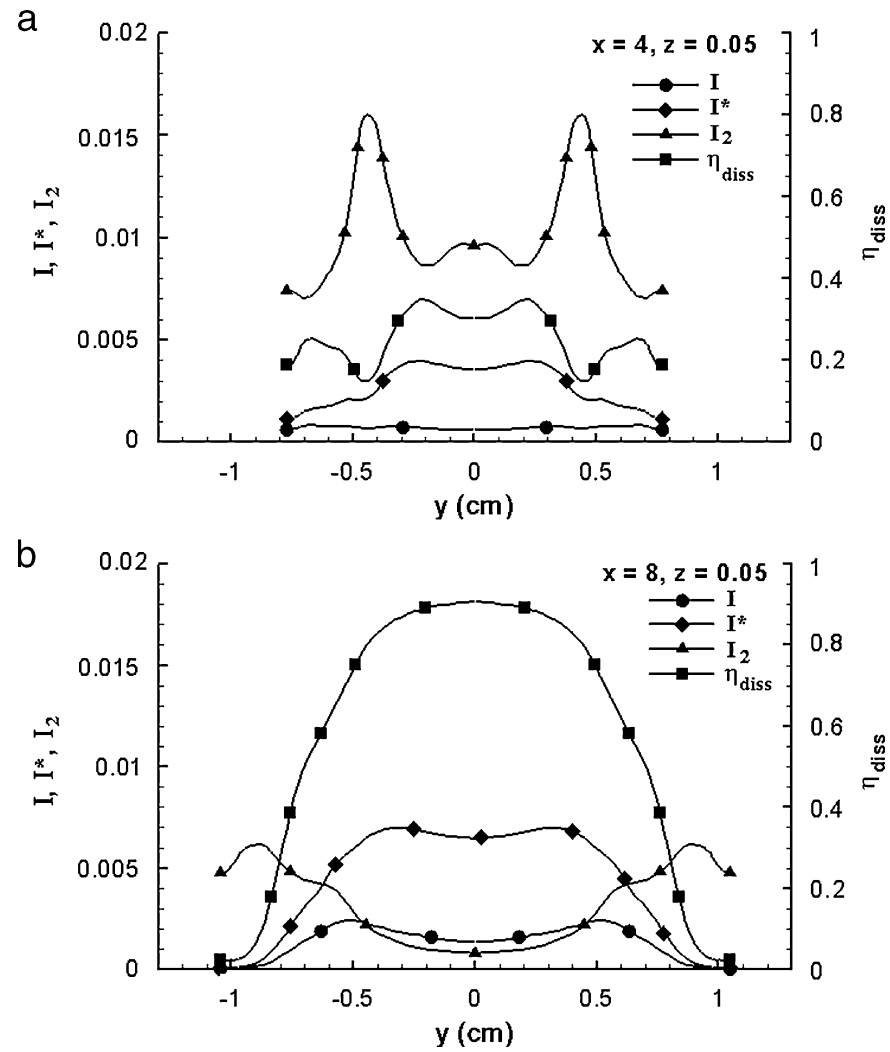

Fig. 9. Spanwise distribution of the non-dimensional densities (normalized by the primary flow density) of $\mathrm{I}_{2}, \mathrm{I}$, and $\mathrm{I}^{*}$ and the dissociation efficiency in different streamwise sections (case (1) $\mathrm{I}_{2} / \mathrm{N}_{2}=1 / 60$ ).

\subsection{Spatial evolution of the small signal gain}

For case (1) with $I_{2} / N_{2}=1 / 60$, the distribution of the small signal gain is plotted in Fig. 6. In Fig. 6a, the small signal gain increases along the streamwise direction and reaches about $0.009 \mathrm{~cm}^{-1}$ in the range of $x=6-7 \mathrm{~cm}$. Downstream the maximum value, the gain decreases slowly to around $0.008 \mathrm{~cm}^{-1}$ at the nozzle exit. The evolutions along four different $z$ planes $(z=0.0,0.033,0.066$, and $0.1 \mathrm{~cm}$ ), as given in Fig. 6a, show that the gain is nearly uniform in the $z$ direction. In Fig. 6b, the gain decreases to almost zero in both boundary layers. The spanwise distribution of the small signal gain becomes relatively smooth within $x=7-9 \mathrm{~cm}$. If we ignore the boundary layer effect, the alternate jet mixing scheme can generate a uniform gain field within a relatively wide range in the nozzle.

In case (2), we set the mole ratio of $\mathrm{I}_{2} / \mathrm{N}_{2}$ to a rich iodine condition of $2 / 59$. The small signal gain appears to be a different spatial evolution. The gain distribution is asymmetrical in the $z$ direction in the near field of jets (where $x<2 \mathrm{~cm}$, as in Fig. 7a) but becomes uniform in the far field. The maximum gain in case (2) is higher than that of case (1). However, the gain decreases more rapidly to a lower value at the nozzle exit. Fig. 7b shows a high gain in the core region of the nozzle flow. At the same time, two negative gain strips along the jet trajectories shorten the 

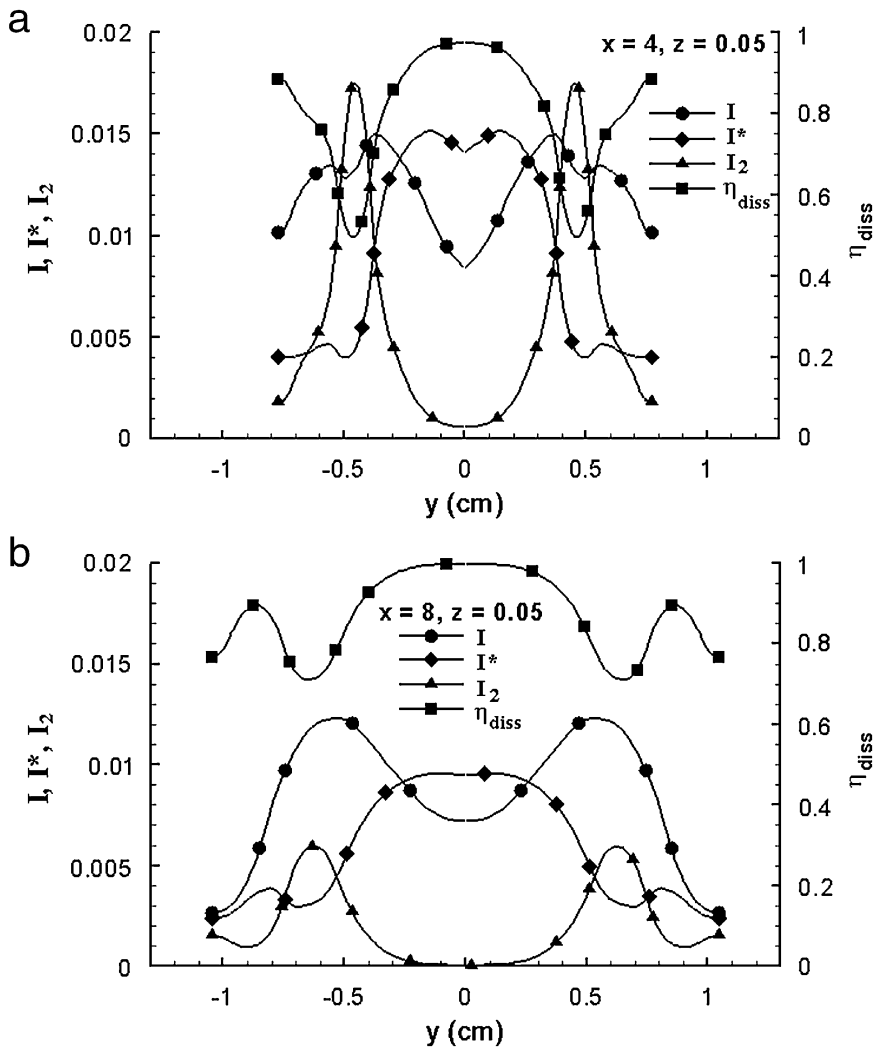

Fig. 10. Spanwise distribution of the non-dimensional densities (normalized by the primary flow density) of $\mathrm{I}_{2}, \mathrm{I}$, and $\mathrm{I}^{*}$ and the dissociation efficiency in different streamwise sections (case (2) $\mathrm{I}_{2} / \mathrm{N}_{2}=2 / 59$ ).

scope of the effective gain distribution in the $y$ direction. The cause of the negative gain is explained later.

In case (3), we set the mole ratio of $I_{2} / \mathrm{N}_{2}$ to a lean iodine condition of 0.5/60.5. As shown in Fig. 8, the gain increases slowly along the streamwise direction. In this condition, reaction (4) becomes the bottleneck of the self-catalyzed chain reaction mechanism, even with relatively superfluous $\mathrm{O}_{2}\left({ }^{1} \Delta\right)$.

The dissociation coefficient of molecular iodine, which is an important parameter that indicates the efficiencies of mixing and chemical reaction in the nozzle flow, is defined as

$\eta_{\text {diss }}=\frac{\left(\rho_{\mathrm{I}}+\rho_{\mathrm{I}^{*}}\right)}{\left(\rho_{\mathrm{I}_{2}}+\rho_{\mathrm{I}}+\rho_{\mathrm{I}^{*}}\right)}$.

Figs. 9 and 10 show the variation of the dissociation coefficient and components such as $\mathrm{I}_{2}$, I, and I* at different streamwise locations for case (1) and (2), respectively. Through Fig. 9a and b, we can see the bi-peak distribution of molecular iodine along the jet trajectories. In addition, the dissociation coefficient is about $90 \%$ at $x=8 \mathrm{~cm}$ in the core of the nozzle flow. Within the bi-peak region and the boundary layers, the dissociation coefficient becomes very low. Furthermore, an abundant of $I^{*}$ is generated in the core flow where the primary flow and the jet flows are mixed sufficiently. The concentration of the ground state iodine atom remains lower than I* throughout the entire flowfield of the nozzle. These features prove that both the dissociation of $\mathrm{I}_{2}$ and the pumping reaction work well. Consequently, positive gain is formed in the core flowfield. In case (2), a large amount of singlet oxygen, $\mathrm{O}_{2}\left({ }^{1} \Delta\right)$, is consumed and one of the members of the chain, I*, is partially quenched for the maintenance and acceleration of the chain reactions. Fig. 10a shows the distribution of some reactants and the dissociation coefficient in the near field of the jets at $x=4 \mathrm{~cm}$, where the dissociation coefficient is already almost $100 \%$ in the core flow. In the flow range where $0.5<|y|<1 \mathrm{~cm}$, the dissociation coefficient varies from about $50 \%$ to $90 \%$, as can be seen in Figs. 10a and b. In this region, however, the available $\mathrm{O}_{2}\left({ }^{1} \Delta\right)$ and then I* are quickly consumed in the dissociation process by the relatively superfluous $\mathrm{I}_{2}$. As a result, more atomic iodine is generated and not enough $\mathrm{O}_{2}\left({ }^{1} \Delta\right)$ is left in the flow to pump them into I*. Consequently, the small signal gain naturally becomes negative within these regions since it is proportional to $\left.\left(\left[\mathrm{I}^{*}\right]\right)-0.5[\mathrm{I}]\right)$.

\section{Conclusion}

Through numerical studies on the mixing and reactive flow in a COIL nozzle that operates without primary buffer gas, we achieved the following conclusions:

(1) A reasonable distribution of the small signal gain can be obtained through an interleaved jet scheme, if there is a proper mole ratio between the molecular iodine and nitrogen in the jets.

(2) A rich iodine condition produces negative gain strips along the jet trajectories, and this phenomenon shortens the effective gain region in the $y$ direction. Furthermore, the small signal gain changes rapidly in the streamwise direction.

(3) A lean iodine condition leads to a low growth rate in the small signal gain.

According to these characteristics of gain evolution, an adjustment of the mole ratio between the molecular iodine and the nitrogen can be used as one accessorial way to control the gain length and gain growth rate, especially when the location of the jet orifices is restricted.

\section{Acknowledgement}

The authors thank Prof. B.G. Wu and Prof. G.W. Yang at the Institute of Mechanics, CAS. This work was supported partially by Korea Research Foundation Grant no. KRF-2005-005-J09901.

\section{References}

[1] Fang BJ, Sang FT, Chen F, et al. Experimental study on kW COIL with nitrogen buffer gases. High Power Laser Particle Beam 2002;14(3):447-50 (in Chinese).

[2] Fang BJ, Sang FT, Chen F, et al. Design and experimental results of $2 \mathrm{~kW}$ COIL with nitrogen buffer gases. High Power Laser Particle Beam 2003;15(12):1148-50 (in Chinese). 
[3] Furman D, Barmashenko BD, Rosenwaks S. Parameter study of an efficient supersonic chemical oxygen-iodine laser/jet generator system operating without buffer gas. IEEE J Quantum Electron 1998;34(7):1068-74.

[4] Furman D, Bruins E, Rybalkin V, et al. Parameter study of smallsignal gain in s slit nozzle, supersonic chemical oxygen-iodine laser operating without primary buffer gas. IEEE J Quantum Electron 2001;37(2):174-82.

[5] Bruins E, Furman D, Rybalkin V, et al. One-dimensional modeling of the gain and temperature in a supersonic chemical oxygen-iodine laser with transonic injection of iodine. IEEE J Quantum Electron 2002;38(4):345-52.

[6] Rybalkin V, Katz A, Furman D, et al. Spatial distribution of the gain and temperature across the flow in a slit-nozzle supersonic chemical oxygen-iodine laser with transonic and supersonic schemes of iodine injection. IEEE J Quantum Electron 2002;38(10):1398-405.

[7] Mikatarian RR, Jumper EJ, Woolhiser CC. Fluid dynamic issues in continuous-wave, short-wavelength chemical laser. AIAA Paper 1988-2748. AIAA Aerospace Science Meeting, Jan. 12-15, 1988, Reno, NV, USA.

[8] Miller JA, Jumper EJ. Role of mixing in the chemical oxygen-iodine laser reactions. AIAA J 1994;32(6):1228-33.
[9] Paschkewitz J, Shang J, Miller J, et al. An assessment of COIL physical property and chemical kinetic modelling methodologies. AIAA Paper 2000-2574. 31st AIAA Plasmadynamics and Lasers Conference, June 19-22, 2000, Denver, CO, USA.

[10] Carroll DL, King DM. Modeling the electriCOIL system. AIAA Paper 2002-2277. 33 Plasmadynamics and Lasers Conference, May 20-23, 2002, Maui, Hawaii, USA.

[11] Carroll DL. Chemical modeling with genetic algorithms. AIAA J 1995;33(8):1454-62.

[12] Eppard WM, McGrory WD, Godfrey AG, et al. Recent advances in numerical techniques for the design and analysis of COIL system. AIAA Paper 2000-2576. 31st AIAA Plasmadynamics and Lasers Conference, June 19-22, 2000, Denver, CO, USA.

[13] Jiang ZL, Takayama K, Chen YS. Dispersion conditions for nonoscillatory shock capturing schemes and its applications. Comp Fluid Dynamics J 1995;4:137-50.

[14] Jiang ZL. On dispersion-controlled principles for non-oscillatory shock-capturing schemes. Acta Mechanica Sinica 2004;20(1):1-15.

[15] Miller JH, Shang JS, Tomaro RF, et al. Computation of COIL nozzle flowfields with transonic injection. AIAA Paper 2000-2575. 31st AIAA Plasmadynamics and Lasers Conference, June 19-22, 2000, Denver, CO, USA. 